\title{
Augmented Eccentric Connectivity Index of Some Thorn Graph
}

\author{
Nilanjan De \\ Department of Basic Science and Humanities (Mathematics), \\ Calcutta Institute of Engineering and Management, Kolkata, India. \\ Email: de.nilanjan@ rediffmail.com
}

\begin{abstract}
The augmented eccentric connectivity index is defined as the summation of the quotients of the product of adjacent vertex degrees and eccentricity of the concerned vertex of a graph which is a generalization of eccentric connectivity index. In this paper we present explicit expressions for the values of augmented eccentric connectivity indices of some particular thorn graphs like thorn path, thorn cycle and thorn star and then consider a particular thorn star, dendrimers.
\end{abstract}

Keywords: Eccentric Connectivity Index, Thorn Graph, Dendrimers.

\section{Introduction}

Let $G$ be any simple connected graph with vertex set $V(G)$ and edge set $E(G)$ and $n$ be the number of vertices. Let the distance between any two vertices of $V(G)$, is equal to the length of the shortest path connecting them. Also for a given vertex of $V(G)$ its eccentricity is the largest distance from that vertex to any other vertices of $G$. The radius and diameter of the graph are the smallest and largest eccentricity among all the vertices of $G$ respectively [1]. The eccentric connectivity index of a graph $\mathrm{G}$ was proposed by Sharma, Goswami and Madan [4] and is defined as

$$
\xi^{c}(G)=\sum_{i=1}^{n} d\left(v_{i}\right) \varepsilon\left(v_{i}\right)
$$

where $d\left(v_{i}\right)$ is the degree i.e. number of first neighbor of $v_{i}$ of $V(G)$ and $\varepsilon\left(v_{i}\right)$ is the eccentricity of the vertex $v_{i}$ of $V(G)$. This topological index is subject to a large 
number of chemical [18] and mathematical [9] studies. A generalization of eccentric connectivity index, known as augmented eccentric connectivity index of a graph $G$ was proposed by Dureja and Madan [5] and is defined as

$$
\xi^{a c}(G)=\sum_{i=1}^{n} \frac{M\left(v_{i}\right)}{\varepsilon\left(v_{i}\right)}
$$

where $M\left(v_{i}\right)$ denotes the product of degrees of all neighbors of vertex $v_{i}$. From above definition it is clear that, as the degrees are taken over the neighborhoods and then multiplied, so the contribution of a vertex to this index is non-local and again since the reciprocal of eccentricity is considered for a vertex so the contribution of a vertex is also non-linear. The study of mathematical as well as chemical properties of this index has started recently (see [2], [3]).

Gutman [8] first proposed the concept of thorn graphs and different applications have been studied by many others. Let $G$ be the parent graph on $n$ vertices $v_{1}, v_{2}, \ldots, v_{n}$ and $\left(p_{1}, p_{2}, \ldots, p_{n}\right)$ be an $n$-tuple on positive integers. Then the thorn graph $G^{*}=G^{*}\left(p_{1}, p_{2}, \ldots, p_{n}\right)$ formed by attaching $p_{i}(\geq 1), i=1,2, \ldots n$ new vertices of degree one to each vertex $v_{i}$ of $G$. Several study of different topological indices of general and some particular thorn graphs and trees like wiener number [10,12], terminal Wiener index [14], modified Wiener index [15], altered Wiener Index [16], Hosoya polynomial [11], Zagreb polynomial [13], Schultz Index [7] and eccentric connectivity index [9] has already been considered.

In this paper we derive some explicit expressions of augmented eccentric connectivity indices of some particular thorn graphs like thorn path, thorn cycle, thorn star and dendrimers either in terms of other topological indices like Zagreb indices and multiplicative Zagreb Indices or recursively express bigger thorn graph in terms of smaller thorn graphs.

\section{Main Results}

\subsection{Thorn Cycle}

Let $C_{n}$ is an $n$ vertex cycle with vertices labeled as $v_{i}$ and $C_{n}{ }^{*}$ is the thorn cycle obtained from $C_{n}$ where each $v_{i}$ consists of $p_{i}$ thorn named as $v_{i j}(i=1,2, \ldots, n$ and $\left.j=1,2, \ldots, p_{i}\right)$. Thus $C_{n}{ }^{*}$ consists of $n$ vertices of degree $\left(p_{i}+2\right)$ and $T$ vertices $v_{i j}$ of degree one, where $T$ is the total number of thorns. The augmented eccentric connectivity index of thorn cycle $C_{n}{ }^{*}$ is obtained as follows:

Theorem 2.1: The augmented eccentric connectivity index of thorn cycle $C_{n}{ }^{*}$ is given by 


$$
\xi^{a c}\left(C_{n}^{*}\right)=\left\{\begin{array}{l}
\frac{2}{n+4}\left[M_{1}\left(C_{n}^{*}\right)-3 T-4 n\right]+\frac{2}{n+2} \sum_{i=1}^{n} M\left(v_{i}\right), \text { when } n \text { is even } \\
\frac{2}{n+3}\left[M_{1}\left(C_{n}^{*}\right)-3 T-4 n\right]+\frac{2}{n+1} \sum_{i=1}^{n} M\left(v_{i}\right), \text { when } n \text { is odd }
\end{array}\right.
$$

where $M\left(v_{i}\right)=\left(p_{j}+2\right)\left(p_{k}+2\right),(i, j) \&(i, k) \in E\left(C_{n}\right)$.

Proof: To calculate augmented eccentric connectivity index of $C_{n}{ }^{*}$ we consider the following two cases:

Case I: When $n$ is an odd number. Then $\varepsilon\left(v_{i}\right)=\frac{n+1}{2}, M\left(v_{i}\right)=\left(p_{j}+2\right)\left(p_{k}+2\right)$

where $(i, j) \&(i, k) \in E\left(C_{n}\right)$. Also $\varepsilon\left(v_{i j}\right)=\frac{(n+3)}{2}$ and $M\left(v_{i j}\right)=p_{i}+2$ for $j=1,2, \ldots, p_{i}$ and $i=1,2, \ldots . n$.

Thus, $\xi^{a c}\left(C_{n}^{*}\right)=\sum_{i=1}^{n} \frac{M\left(v_{i}\right)}{\varepsilon\left(v_{i}\right)}+\sum_{i=1}^{n} \sum_{j=1}^{p_{i}} \frac{M\left(v_{i j}\right)}{\varepsilon\left(v_{i j}\right)}$

$$
\begin{aligned}
& =\frac{2}{n+1} \sum_{i=1}^{n} M\left(v_{i}\right)+\frac{2}{n+3} \sum_{i=1}^{n} p_{i}\left(p_{i}+2\right) \\
& =\frac{2}{n+1} \sum_{i=1}^{n} M\left(v_{i}\right)+\frac{4}{n+3} \sum_{i=1}^{n} p_{i}+\frac{2}{n+3} \sum_{i=1}^{n} p_{i}^{2}
\end{aligned}
$$

Again since, $M_{1}\left(C_{n}^{*}\right)=\sum_{i=1}^{n} p_{i}^{2}+5 T+4 n$, thus from above we get the desired result.

Case II: When $n$ is an even number. Then, similarly $\varepsilon\left(v_{i}\right)=\frac{n+2}{2}$,

$M\left(v_{i}\right)=\left(p_{j}+2\right)\left(p_{k}+2\right)$ where $(i, j) \&(i, k) \in E\left(C_{n}\right)$. Also $\varepsilon\left(v_{i j}\right)=\frac{(n+4)}{2}$ and $M\left(v_{i j}\right)=p_{i}+2$ for $j=1,2, \ldots, p_{i}$ and $i=1,2, \ldots . n$. So proceeding similarly we get the desired result (1).

Corollary 2.1: For t-thorny ring $C_{n . t}$ (having $n$ ring as parent and $t-2$ thorns at each vertex) [10] the augmented eccentric connectivity index is given by

$$
\xi^{a c}\left(C_{n, t}\right)=\left\{\begin{array}{l}
\frac{4 n t}{n+4}\left[\left(\frac{n+2}{n+1}\right) t-1\right] \text { when } n \text { is even } \\
\frac{4 n t}{n+4}\left[\left(\frac{n+2}{n+1}\right) t-1\right] \text { when } n \text { is odd }
\end{array}\right.
$$




\subsection{Thorn Path}

Let $P_{m} *$ be a thorn path obtained by attaching $(a-2)$ pendent vertices to each non pendent vertices of a path $P_{m}$. Now to calculate augmented eccentric connectivity index of a thorn path first we consider the augmented eccentric connectivity index of a path $P_{m}$.

Theorem 2.3: [17] If $H_{m}=\sum_{i=1}^{m} \frac{1}{i}$, the augmented eccentric connectivity index of a path $P_{m}$ with $m$ vertices is given by

$$
\xi^{a c}\left(P_{m}\right)=\left\{\begin{array}{l}
8\left(H_{m-1}-H_{\frac{m-2}{2}}\right)-4\left(\frac{1}{m-1}+\frac{1}{m-2}\right), \text { when } m \text { is even } \\
8\left(H_{m-1}-H_{\frac{m-3}{2}}\right)-4\left(\frac{3}{m-1}+\frac{1}{m-2}\right), \text { when mis odd }
\end{array}\right.
$$

The above result can also be written as

$$
\xi^{a c}\left(P_{m}\right)=\left\{\begin{array}{l}
8 \sum_{i=0}^{n-1} \frac{1}{n+2+i}+4\left(\frac{1}{2 n+2}+\frac{1}{2 n+3}\right), \text { when } m=2 n+4 \text { that is even } \\
8 \sum_{i=1}^{n-1} \frac{1}{n+1+i}+4\left(\frac{1}{2 n+1}+\frac{3}{2 n+2}\right), \text { when } m=2 n+3 \text { that is odd }
\end{array}\right.
$$

Now using these results we find the augmented eccentric connectivity index of thorn path $P_{m}{ }^{*}$ in terms of the augmented eccentric connectivity index of path $P_{m}$.

Theorem 2.4: The augmented eccentric connectivity index of the thorn path $P_{m}{ }^{*}$ is given by

$$
\xi^{a c}\left(P_{m}^{*}\right)=\left\{\begin{array}{l}
\frac{1}{2} a(a-1) \xi^{a c}\left(P_{m}\right)-\frac{4 a(a-2)}{m}, \text { when } m \text { is even } \\
\frac{1}{2} a(a-1) \xi^{a c}\left(P_{m}\right)-\frac{4 a(a-2)}{m+1}, \text { when mis odd }
\end{array}\right.
$$

Proof: To poof this result we consider the following two cases.

Case: $I$ Let $P_{m}^{*}$ be a thorn path obtained from a path $P_{m}$ on even number of vertices, so that $m=2 n+4$. Also let the thorn tree $P_{2 n+4}^{*}$ is obtained by attaching (a-2) pendent vertices to the non pendent vertices of $P_{2 n+4}$. Let the vertices of $P_{2 n+4}$ labeled as $v_{n+1}^{\prime}, v_{n}^{\prime}, v_{n-1}^{\prime}, \ldots . v_{2}^{\prime}, v_{1}^{\prime}, v_{0}^{\prime}, v_{0}, v_{1}, v_{2}, \ldots, v_{n-1}, v_{n}, v_{n+1}$ where $v_{0}^{\prime}$ and $v_{0}$ are centers of the parent path so that for $P_{2 n+4}^{*}$ we have $M\left(v_{i}\right)=a^{2}=M\left(v_{i}^{\prime}\right)$ for $i=0,1,2, \ldots, n-1$ and $M\left(v_{j}\right)=a=M\left(v_{j}^{\prime}\right)$ for $j=n, n+1$. Again for $P_{2 n+4}^{*}$ we have $\varepsilon\left(v_{i}\right)=n+2+i=\varepsilon\left(v_{i}^{\prime}\right)$ for $i=0,1,2, \ldots, n+1$.

Again, let the newly attached pendent vertices to the vertices $v_{i}$ and $v_{i}^{\prime}$ of $P_{2 n+4}$, are denoted by $l_{i j}$ and $l_{i j}^{\prime}$ for $i=0,1,2, \ldots, n$ and $j=1,2, \ldots,(a-2)$. Thus 
$\varepsilon\left(l_{i j}\right)=\varepsilon\left(v_{i}\right)+1=\varepsilon\left(v_{i}^{\prime}\right)+1=\varepsilon\left(l_{i j}^{\prime}\right)$ for $i=0,1,2, \ldots, n$ and $j=1,2, \ldots,(a-2)$. Similarly $M\left(l_{i j}\right)=a=M\left(l_{i j}^{\prime}\right)$ for $i=0,1,2, \ldots, n$ and $j=1,2, \ldots,(a-2)$.

Thus the augmented eccentric connectivity index of $P_{2 n+4}^{*}$ is given by

$$
\xi^{a c}\left(P_{2 n+4}^{*}\right)=\xi_{1}^{a c}\left(P_{2 n+4}^{*}\right)+\xi_{2}^{a c}\left(P_{2 n+4}^{*}\right)
$$

Where, $\xi_{1}^{a c}\left(P_{2 n+4}^{*}\right)=\sum_{i=0}^{n+1} \frac{M\left(v_{i}\right)}{\varepsilon\left(v_{i}\right)}+\sum_{i=0}^{n+1} \frac{M\left(v_{i}^{\prime}\right)}{\varepsilon\left(v_{i}^{\prime}\right)}$

and $\xi_{2}^{a c}\left(P_{2 n+4}^{*}\right)=\sum_{i=0}^{n} \sum_{j=1}^{a-2} \frac{M\left(l_{i j}\right)}{\varepsilon\left(l_{i j}\right)}+\sum_{i=0}^{n} \sum_{j=1}^{a-2} \frac{M\left(l_{i j}^{\prime}\right)}{\varepsilon\left(l_{i j}^{\prime}\right)}$

Now $\xi_{1}^{a c}\left(P_{2 n+4}^{*}\right)=2\left[\sum_{i=0}^{n-1} \frac{M\left(v_{i}\right)}{\varepsilon\left(v_{i}\right)}+\sum_{j=n}^{n+1} \frac{M\left(v_{j}\right)}{\varepsilon\left(v_{j}\right)}\right]=2\left[\sum_{i=0}^{n-1} \frac{a^{2}}{n+2+i}+\sum_{j=n}^{n+1} \frac{a}{(n+2+j)}\right]$

$$
\begin{aligned}
& =2 a^{2}\left\{\frac{1}{8} \xi^{a c}\left(P_{2 n+4}\right)-\frac{1}{2}\left(\frac{1}{2 n+2}+\frac{1}{2 n+3}\right)\right\}+2 a\left\{\frac{1}{2 n+2}+\frac{1}{2 n+3}\right\} \\
& =\frac{a^{2}}{4} \xi^{a c}\left(P_{2 n+4}\right)+a(a-2)\left(\frac{1}{2 n+2}+\frac{1}{2 n+3}\right)
\end{aligned}
$$

Again, $\xi_{2}^{a c}\left(P_{2 n+4}^{*}\right)=\sum_{i=0}^{n} \sum_{j=1}^{a-2} \frac{a}{\varepsilon\left(v_{i}\right)+1}+\sum_{i=0}^{n} \sum_{j=1}^{a-2} \frac{a}{\varepsilon\left(v_{i}^{\prime}\right)+1}=2 a(a-2) \sum_{i=0}^{n} \frac{1}{(n+3+i)}$

$$
=a(a-2)\left[\frac{1}{4} \xi^{a c}\left(P_{2 n+3}\right)+\left(\frac{1}{2 n+2}+\frac{1}{2 n+3}\right)-\frac{2}{n+2}\right]
$$

Thus from (3) $\xi^{a c}\left(P_{2 n+4}^{*}\right)=\frac{1}{2} a(a-1) \xi^{a c}\left(P_{2 n+4}\right)-\frac{2 a(a-2)}{n+2}$

and hence the desired result follows.

Case: II Let $P_{m}^{*}$ be a thorn path obtained from a path $P_{m}$ on odd number of vertices, so that $m=2 n+3$. Also let the thorn tree $P_{2 n+3}^{*}$ is obtained by attaching ( $a$-2) pendent vertices to the non pendent vertices of $P_{2 n+3}$. Let the vertices of $P_{2 n+3}$ labeled as $v_{n+1}^{\prime}, v_{n}^{\prime}, v_{n-1}^{\prime}, \ldots . v_{2}^{\prime}, v_{1}^{\prime}, v_{0}, v_{1}, v_{2}, \ldots, v_{n-1}, v_{n}, v_{n+1}$, where $v_{0}$ is the center of the parent path so that for $P_{2 n+3}^{*}$ we have $M\left(v_{0}\right)=a^{2}, M\left(v_{i}\right)=a^{2}=M\left(v_{i}^{\prime}\right)$ for $i=1,2, \ldots, n-1$ and $M\left(v_{j}\right)=a=M\left(v_{j}^{\prime}\right)$ for $j=n, n+1$. Again for $P_{2 n+3}^{*}$ we have $\varepsilon\left(v_{0}\right)=n+1, \varepsilon\left(v_{i}\right)=n+1+i=\varepsilon\left(v_{i}^{\prime}\right)$ for $i=1,2, \ldots, n+1$.

Again, let the newly attached pendent vertices to the vertices $v_{0}, v_{i}$ and $v_{i}^{\prime}$ of $P_{2 n+3}$, are denoted by $l_{0 \mathrm{j}}, l_{i j}$ and $l_{i j}^{\prime}$ for $i=0,1,2, \ldots, n$ and $j=1,2, \ldots,(a-2)$. Thus $\varepsilon\left(l_{i j}\right)=\varepsilon\left(v_{i}\right)+1=\varepsilon\left(v_{i}^{\prime}\right)+1=\varepsilon\left(l_{i j}^{\prime}\right)$ for $i=0,1,2, \ldots, n$ and $j=1,2, \ldots,(a-2)$. Similarly $M\left(l_{i j}\right)=a=M\left(l_{i j}^{\prime}\right)$ for $i=0,1,2, \ldots, n$ and $j=1,2, \ldots,(a-2)$.

Thus the augmented eccentric connectivity index of $P_{2 n+4}^{*}$ is given by 


$$
\xi^{a c}\left(P_{2 n+4}^{*}\right)=\xi_{1}^{a c}\left(P_{2 n+4}^{*}\right)+\xi_{2}^{a c}\left(P_{2 n+4}^{*}\right)
$$

where

$$
\begin{aligned}
& \xi_{1}^{a c}\left(P_{2 n+3}^{*}\right)=\frac{M\left(v_{0}\right)}{\varepsilon\left(v_{0}\right)}+\sum_{i=1}^{n+1} \frac{M\left(v_{i}\right)}{\varepsilon\left(v_{i}\right)}+\sum_{i=1}^{n+1} \frac{M\left(v_{i}^{\prime}\right)}{\varepsilon\left(v_{i}^{\prime}\right)}=\frac{a^{2}}{4} \xi^{a c}\left(P_{2 n+4}\right)-a(1-a)\left(\frac{1}{2 n+1}+\frac{1}{2 n+2}\right) \\
& \text { and } \xi_{2}^{a c}\left(P_{2 n+3}^{*}\right)=\sum_{j=0}^{a-2} \frac{M\left(l_{0 j}\right)}{\varepsilon\left(l_{0 j}\right)}+\sum_{i=1}^{n} \sum_{j=1}^{a-2} \frac{M\left(l_{i j}\right)}{\varepsilon\left(l_{i j}\right)}+\sum_{i=1}^{n} \sum_{j=1}^{a-2} \frac{M\left(l_{i j}^{\prime}\right)}{\varepsilon\left(l_{i j}^{\prime}\right)} \\
& =a(a-2)\left[\frac{1}{4} \xi^{a c}\left(P_{2 n+3}\right)+\left(\frac{1}{2 n+1}+\frac{1}{2 n+2}\right)-\frac{2}{n+2}\right]
\end{aligned}
$$

So proceeding similarly we get the desired result as stated above.

Also from Theorem 2.3 and Theorem 2.4 the augmented eccentric connectivity index of thorn path $P_{m}{ }^{*}$ in terms of $H_{m}$ is given by

Theorem 2.5: The augmented eccentric connectivity index of the thorn path $P_{m}{ }^{*}$ is given by

$$
\xi^{a c}\left(P_{m}^{*}\right)=\left\{\begin{array}{r}
2 a(a-1)\left[2\left(H_{m-1}-H_{\frac{m-2}{2}}\right)-\left(\frac{1}{m-1}+\frac{1}{m-2}-\frac{2}{m}\right)\right]+\frac{4 a}{m}, \text { when } m \text { is even } \\
2 a(a-1)\left[2\left(H_{m-1}-H_{\frac{m-3}{2}}\right)-\left(\frac{3}{m-1}+\frac{1}{m-2}-\frac{2}{m+1}\right)\right]+\frac{4 a}{m+1}, \text { when } m \text { is odd }
\end{array}\right.
$$

\subsection{Thorn Star}

The thorn star $K_{1, n}^{*}$ with parameters with parameters $p_{i}, i=1,2, \ldots n$, is the graph obtained from the star $K_{1, n}$ by adding $p_{i}$ pendent vertices to the $i$ th pendent vertex of $K_{1, n}$. Let the center of the star is denoted by $c$ and the all the remaining pendent vertices are denoted by $v_{i}$ so that $M(c)=\varepsilon(c)=1, M\left(v_{i}\right)=n$ and $\varepsilon\left(v_{i}\right)=2$. Thus, $\xi^{a c}\left(K_{1, n}\right)=\left(1+\frac{n^{2}}{2}\right)$.

Theorem 2.6: The augmented eccentric connectivity index of thorn star $K_{1, n}^{*}$ is given by 


$$
\xi^{a c}\left(K_{1, n}^{*}\right)=\frac{1}{2 n} \sqrt{\prod_{1}\left(K_{1, n}^{*}\right)}+\frac{1}{4} M_{1}\left(K_{1, n}^{*}\right)-\frac{1}{4} T+\frac{n}{4}\left(\frac{n}{3}-1\right)
$$

Proof: Let the newly attached pendent vertices are denoted by $v_{i j}$ so that in $K_{1, n}^{*}$, $M(c)=\prod_{i=1}^{n}\left(p_{i}+1\right), \varepsilon(c)=2, M\left(v_{i}\right)=n, \varepsilon\left(v_{i}\right)=3$ and $M\left(v_{i j}\right)=p_{i}+1, \varepsilon\left(v_{i j}\right)=4$ for $j=1,2, \ldots, p_{i}$ and $i=1,2, \ldots . n$. Thus the eccentric connectivity index of thorn star is given by

$$
\begin{aligned}
\xi^{a c}\left(K_{1, n}^{*}\right) & =\frac{M(c)}{\varepsilon(c)}+\sum_{i=1}^{n} \frac{M\left(v_{i}\right)}{\varepsilon\left(v_{i}\right)}+\sum_{i=1}^{n} \sum_{j=1}^{p_{i}} \frac{M\left(v_{i j}\right)}{\varepsilon\left(v_{i j}\right)} \\
& =\frac{1}{2} \prod_{i=1}^{n}\left(p_{i}+1\right)+\frac{n^{2}}{3}+\sum_{i=1}^{n} \sum_{j=1}^{p_{i}} \frac{p_{i}+1}{4} \\
& =\frac{1}{2} \sqrt{\prod_{1}\left(K_{1, n}^{*}\right)}+\frac{n^{2}}{3}+\frac{T}{4}+\sum_{i=1}^{n} p_{i}^{2}
\end{aligned}
$$

where the first multiplicative Zagreb index of $G$ is defined as [6],

$\prod_{1}(G)=\prod_{i \in V(G)} d\left(v_{i}\right)^{2}$. Now since the first Zagreb index of $K_{1, n}^{*}$ is given by

$M_{1}\left(K_{1, n}^{*}\right)=\sum_{i=1}^{n} p_{i}^{2}+2 T+n^{2}+n$ so the desired result follows from above.

Corollary 2.2: The augmented eccentric connectivity index of $S_{k, t}$ is given by

$$
\xi^{a c}\left(S_{k, t}\right)=\frac{4 n t}{n+1}\left[\frac{(t-2)(n+2)}{n+3}+1\right]
$$

where, the thorn star $S_{k, t}$ (as in [10]) is obtained from a star with $(k+1)$ vertices by joining $(t-1)$ pendent vertices to each $v_{i}(i=1,2, . ., k)$.

\subsection{Dendrimers}

Let the monocentric regular dendrimers is denoted by $T_{d, k}$ so that the centre $v_{0}$ and the all the other non-pendent vertices is of degree $d$ and the distance of every pendent vertices from the centre $v_{0}$ is $k$ that is $k$ denotes the diameter of $T_{d, k}$. Obviously the number of vertices of $T_{d, k}$ is $1+\frac{d\left[(d-1)^{k}-1\right]}{d-2}$ so that $T_{d, k}$ consists of $d(d-1)^{k}$ number of thorns. Thus we can also say that there is total $d(d-1)^{p-1}$ number of vertices whose distance from the centre $v_{0}$ is $p$.

Theorem 2.7: The augmented eccentric connectivity index of regular monocentric dendrimers is recursively expressed as

$$
\xi^{a c}\left(T_{d, k}\right)=\frac{1}{d-1} \xi^{a c}\left(T_{d, k-1}\right)+\frac{d^{2}(d-1)^{k-2}(2 k d-d+1)}{2 k(2 k-1)}-\frac{d^{d}(2 k-1)}{k(k-1)(d-1)}+
$$




$$
\frac{d\left(d^{d}-d\right)(d-1)^{k-4}(2 k d-3 d+1)}{2(k-1)(2 k-3)}
$$

where $d$ is the degree of the non pendent vertices and $k$ is the diameter of the tree.

Proof: Let the vertices whose distance from the centre is $p$ be denoted by $v_{p j}$. Clearly the eccentricity of the central vertex $v_{o}$ is $k$ i.e. $\varepsilon\left(v_{0}\right)=k$ and $\varepsilon\left(v_{i j}\right)=k+i$ where $v_{i j}$ are the vertices whose distance from $v_{0}$ is $i$. Also $M\left(v_{0}\right)=d^{d}$ for $i=1,2, \ldots, k-2$ and $M\left(v_{i j}\right)=d^{d}$ for $i=1,2, \ldots, k-2$ and $M\left(v_{i j}\right)=d$ for $i=k-1 \& k$, $j=1,2, \ldots, d(d-1)^{i-1}$.

Thus the augmented eccentric connectivity index of $T_{d, k}$ is given by

$$
\begin{aligned}
\xi^{a c}\left(T_{d, k}\right) & =\frac{M\left(v_{0}\right)}{\varepsilon\left(v_{0}\right)}+\sum_{i=1}^{k-2} \sum_{j=1}^{d(d-1)^{i-1}} \frac{M\left(v_{i j}\right)}{\varepsilon\left(v_{i j}\right)}+\sum_{i=k-1}^{k} \sum_{j=1}^{d(d-1)^{i-1}} \frac{M\left(v_{i j}\right)}{\varepsilon\left(v_{i j}\right)} \\
& =\frac{d^{d}}{k}+\sum_{i=1}^{k-2} \frac{d^{d} d(d-1)^{i-1}}{k+i}+\sum_{j=1}^{d(d-1)^{k-2}} \frac{d}{2 k-1}+\sum_{j=1}^{d(d-1)^{k-1}} \frac{d}{2 k} \\
& =\frac{d^{d}}{k}+\frac{d^{2}(d-1)^{k-2}}{2 k-1}+\frac{d^{2}(d-1)^{k-1}}{2 k}+\sum_{i=1}^{k-2} \frac{d^{d+1}(d-1)^{i-1}}{k+i}
\end{aligned}
$$

From above it is clear that,

$$
\xi^{a c}\left(T_{d, k-1}\right)=\frac{d^{d}}{k-1}+\frac{d^{2}(d-1)^{k-3}}{2 k-3}+\frac{d^{2}(d-1)^{k-2}}{2 k-2}+\sum_{i=1}^{k-3} \frac{d^{d+1}(d-1)^{i-1}}{k-1+i}
$$

Now let, $S_{1}=\sum_{i=1}^{k-2} \frac{d^{d+1}(d-1)^{i-1}}{k+i}$ and $S_{2}=\sum_{i=1}^{k-3} \frac{d^{d+1}(d-1)^{i-1}}{k-1+i}$ so that

$$
S_{1}=\frac{1}{d-1} S_{2}+d^{d+1}\left[\frac{(d-1)^{k-4}}{2 k-3}+\frac{(d-1)^{k-3}}{2 k-2}-\frac{(d-1)^{-1}}{k}\right]
$$

So we have

$$
S_{1}=\frac{1}{d-1} \xi_{2}^{a c}\left(T_{d, k-1}\right)+d\left(d^{d}-1\right)\left[\frac{(d-1)^{k-4}}{2 k-3}+\frac{(d-1)^{k-3}}{2 k-2}\right]+\frac{d^{d}(k+k d-d)}{k(d-1)(k-1)}
$$

Then from above we get

$$
\begin{aligned}
& \xi^{a c}\left(T_{d, k}\right)=S_{1}+\frac{d^{d}}{k}+\frac{d^{2}(d-1)^{k-2}}{2 k-1}+\frac{d^{2}(d-1)^{k-1}}{2 k}+\frac{d^{2}(d-1)^{k-1}}{2 k} \\
= & \frac{1}{d-1} \xi_{2}^{a c}\left(T_{d, k-1}\right)+d\left(d^{d}-1\right)\left[\frac{(d-1)^{k-4}}{2 k-3}+\frac{(d-1)^{k-3}}{2 k-2}\right]+\frac{d^{d}(k+k d-d)}{k(d-1)(k-1)}+\frac{d^{d}}{k}+\frac{d^{2}(d-1)^{k-2}}{2 k-1}
\end{aligned}
$$

Now after some calculations we get the desired result. 


\section{Conclusion}

Since the augmented eccentric connectivity index is based on both non-local and non-linear contributions of vertices, so it difficult to calculate some explicit relations of this index for generalized thorn graphs (like [9]). In this paper we have presented some explicit mathematical expressions of the augmented eccentric connectivity index of some particular thorn graphs. In Theorem 1 the augmented eccentric connectivity index of a thorn cycle is expressed as first Zagreb index of thorn cycle and sum of product of degree of all neighbors of vertices of $C_{n}$. Also in Theorem 4 first the augmented eccentric connectivity index of thorn path is expressed in terms of its parent path and then in Theorem 5 it is expressed in terms of $H_{m}$. From Theorem 6 it is clear that there is a nonlinear relation between the augmented eccentric connectivity index, first Zagreb index and first multiplicative Zagreb index of thorn stars. Also using the recursive relation (9), for a particular value of $d$, we can obtain a series of augmented eccentric connectivity index for monocentric dendrimers. For example when $d=3$, for $k=2,3,4, \ldots$ we get the series $\{21,38.85,64.09, \ldots$.$\} . However, our results still$ leave much to be desired. These results can be extended to generalized thorn graph where some $p_{i}$ are equal to zero.

\section{References}

[1] P. Dankelmann, W. Goddard, C.S. Swart, The average eccentricity of a graph and its subgraphs, Util. Math., 65 (2004), 41-51.

[2] T. Došlić, M. Saheli, Augmented Eccentric Connectivity Index, Miskolc Math. Notes., 12(2) (2011),149-157.

[3] Z. Yarahmadi, Eccentric Connectivity and Augmented Eccentric Connectivity Indices of N-Branched Phenylacetylenes Nanostar Dendrimers, Iranian J. Math. Chem., 1(2) (2010), 105-110.

[4] V. Sharma, R. Goswami, A. K. Madan, Eccentric connectivity index: A novel highly discriminating topological descriptor for structure-property and structure-activity studies, J. Chem. Inf. Model, 37 (1997), 273-282.

[5] H. Dureja, A. K. Madan, Superaugmented eccentric connectivity indices: new generation highly discriminating topological descriptors for QSAR/QSPR modeling, Med. Chem. Res., 16 (2007), 331-341.

[6] I. Gutman, Multiplicative Zagreb Indices of Trees, Bull. Soc. Math. Banja Luka. 18 (2011), 17-23.

[7] D. Vukipeviü, S. Nikoliü, and N. Trinajstiü ,On the Schultz Index of Thorn Graphs, Internet Electron. J. Mol. Des., 4 (2005), 501-514.

[8] I. Gutman, Distance in thorny graph, Publ. Inst. Math. (Beograd,) 63 (1998), 31-36. 
[9] N. De, On eccentric connectivity index and polynomial of thorn graph, Applied Mathematics, 3 (2012), 931-934.

[10] D. Bonchev, D. J. Klein, On the Wiener number of thorn trees, stars, rings, and rods, Croat. Chem. Acta., 75 (2002), 613-620.

[11] H. B. Walikar, H. S. Ramane, L. Sindagi, S. S. Shirakol, I. Gutman, Hosoya polynomial of thorn trees, rods, rings, and stars, Kragujevac J. Sci., 28 (2006), 47-56.

[12] B. Zhou, D. Vukičević, On Wiener-type polynomials of thorn graphs, $J$. Chemometrics, 23(12) (2009), 600-604.

[13] S. Li, Zagreb polynomials of thorn graphs, Kragujevac J. Sci., 33, (2011), 33-38.

[14] A. Heydari , I. Gutman, On the terminal Wiener index of thorn graphs, Kragujevac J. Sci.,32 (2010), 57-64.

[15] B. Zhou, On modified Wiener indices of thorn trees, Kragujevac J. Math., 27 (2005), 5-9.

[16] D. Vukičević , B. Zhou and N. Trinajstić, Altered Wiener Indices of Thorn Trees, Croat. Chem. Acta. , 80 (2007), 283-285.

[17] J. Sedlar, On augmented eccentric connectivity index of graphs and trees, MATCH Commun. Math. Comput. Chem. 68 (2012) 325-342.

[18] J. Yang, F. Xia, The Eccentric Connectivity Index of Dendrimers, Int. J. Contemp. Math. Sciences, 5(45) (2010), 2231 -2236. 